\title{
Toward Applications of Linear Control Systems on the Real World and Theoretical Challenges
}

\author{
Víctor Ayala ${ }^{1, *}$, María Torreblanca ${ }^{2}$ and William Valdivia ${ }^{1}$ \\ 1 Instituto de Alta Investigación, Universidad de Tarapacá, Casilla 7D, Arica 1000000, Chile; \\ wvaldiviah@gmail.com or wvaldiviah@unsa.edu.pe \\ 2 Departamento Académico de Matemáticas, Universidad Nacional de San Agustín de Arequipa, \\ Calle Santa Catalina, Nro. 117, Arequipa 04001, Peru; mtorreblancat@unsa.edu.pe \\ * Correspondence: vayala@uta.cl
}

check for updates

Citation: Ayala, V.; Torreblanca, M.; Valdivia, W. Toward Applications of Linear Control Systems on the Real World and Theoretical Challenges. Symmetry 2021, 13, 167. https:/ / doi.org/10.3390/sym13020167

Academic Editor: Kok Lay Teo Received: 16 December 2020 Accepted: 29 December 2020 Published: 22 January 2021

Publisher's Note: MDPI stays neutral with regard to jurisdictional clai$\mathrm{ms}$ in published maps and institutional affiliations.

Copyright: (C) 2021 by the authors. Licensee MDPI, Basel, Switzerland. This article is an open access article distributed under the terms and conditions of the Creative Commons Attribution (CC BY) license (https:// creativecommons.org/licenses/by/ $4.0 /)$.

\begin{abstract}
Many significant real world challenges arise as optimization problems on different classes of control systems. In particular, ordinary differential equations with symmetries. The purpose of this review article is twofold. First, we give the information we have about the class of Linear Control Systems $\Sigma_{G}$ on a low dimension matrix Lie group G. Second, we invite the Mathematical community to consider possible applications through the Pontryagin Maximum Principle for $\Sigma_{G}$. In addition, we challenge some theoretical open problems. The class $\Sigma_{G}$ is a perfect generalization of the classical Linear Control System on the Abelian group $\mathbb{R}^{n}$. Let $G$ be a Lie group of dimension two or three. Related to $\Sigma_{G}$, this review describes the actual results about controllability, the time-optimal Hamiltonian equations and, the Pontryagin Maximum Principle. We show how to build $\Sigma_{G}$, through several examples on low dimensional matrix groups.
\end{abstract}

Keywords: linear control systems; matrix lie groups; controllability; pontryagin maximum principle

MSC: 93C10; 49J15; 34A26

\section{Introduction}

Many significant real world challenges arise as optimization problems on different classes of control systems. In particular, ordinary differential equations with symmetries. The purpose of this review article is twofold. First, we give the information we have about the class of Linear Control Systems $\Sigma_{G}$ on a low dimension matrix Lie group G. Second, we invite the Mathematical community to consider possible applications through the Pontryagin Maximum Principle for $\Sigma_{G}$. In addition, we challenge some theoretical open problems. The class $\Sigma_{G}$ is a perfect generalization of the classical Linear Control System on the Abelian group $\mathbb{R}^{n}$.

Lie theory refers to the theory integrating the concept of Lie group and Lie algebra through the exponential map, and provides a natural model for the notion of continuous symmetry. Lie groups are widely used in almost every part of modern mathematics and physics. The relevance of the Lie theory in applications has become evident. With respect to this article, we mention that Lie theory is a powerful tool for the study of differential equations $[1,2]$. Therefore, the extension of linear system from $\mathbb{R}^{n}$ to any connected Lie group, it is necessary.

In order to build examples, in this review we explicitly show the Lie algebras of dimension 2 and 3, and its corresponding simply connected Lie groups. Furthermore, we compute all the possible linear vector fields, and the left invariant vector fields. They are the main ingredients to build the dynamic of $\Sigma_{G}$. For both dimensions, we characterize the Linear Algebra Rank Condition $L A R C$, the controllability property, the existence, uniqueness, and the shape of control sets in dimension 2. There is no information about control sets for $\Sigma_{G}$ on dimension 3. From the optimization point of view, we give explicitly 
the Hamiltonian equations of a time-optimal problem for $\Sigma_{G}$, as appear in [3,4]. The optimal quadratic problem is open for this class.

It is very well known that control systems, particularly the classical class $\Sigma_{\mathbb{R}^{n}}$, had been used as a model for beautiful and concrete applications. For instance, optimal control problems: in Economic growth [5], in Mechanics [2,6], in Medicine, Biology and Chemistry in [7,8], in spacecraft [9], in engineering systems in [10], for the Dubin's problem in [11], for the brachistochrone and related topics in [12], etc. In addition, we are confident that the same is true for $\Sigma_{G}$. There are some reasons to believe that. In the first place, the notion of the Lie group allows discovering the symmetries of analytical structures. In addition, it is able to discover the symmetries of classes of differential equations, [13]. Examples of these manifolds are the Abelian group $\mathbb{R}^{n}$, the spheres $S^{n} \subset \mathbb{R}^{n+1}$ for $n=1,3$ and 7 , the set $G L(n, \mathbb{R})$ of the invertible real matrices of order $n$, and its relevant subgroups $S L(n, \mathbb{R})$ of matrices with determinant 1 , the orthogonal group $O(n, \mathbb{R})$, the spinor group $\operatorname{Spin}(n, \mathbb{R})$, the unitary group $U(n, \mathbb{R})$ and many others. These apply not just to real, but also complex coefficients. However, the main reason comes from the Jouan Equivalence Theorem [14] which roughly says that: "Any affine control systems on an arbitrary differentiable manifold is equivalent to a linear control system on a Lie group, or on a homogeneous space if and only if the Lie algebra generated by the vector fields of the system is finite-dimensional".

To understand the meaning of the Equivalence Theorem, and also as a general motivation, consider a tumor growth with initial condition $x_{0}$ and dynamics determined by a vector field $f$ on the space state $M$, as the solution $x(t)$ of the associated differential equation,

$$
x(t)=f(x(t)), x(t) \in M, \text { with } x(0)=x_{0} .
$$

Deciding which combination of treatments is right for a patient is critical. The introduction of the treatments $g^{1}, \ldots, g^{m}$, and the control function $u=\left(u_{1}, \ldots, u_{m}\right) \in \mathcal{U}$ define an affine control system which changes the behavior of the tumor, as the solutions of the controlled family of differential equations

$$
\Sigma_{M}: \dot{x}(t)=f(x(t))+\sum_{j=1}^{m} u_{j}(t) g^{j}(x(t)), x(t) \in M, u \in \mathcal{U}, x(0)=x_{0} .
$$

Here, $\mathcal{U}$ is the admissible class of control functions to be chosen.

This process gives you a way to combine a global tumor treatment in time. Two theoretical-practical problems appear:

To compute $\mathcal{A}\left(x_{0}\right) \subset M$, the reachable set from $x_{0}$ through the controls $u \in \mathcal{U}$, in positive time

Assume $x_{1} \in \mathcal{A}\left(x_{0}\right)$. Starting at $x_{0}$, is it possible to reach $x_{1}$ with the minimum time, or with the minimum collateral damage?

The system $\Sigma_{M}$ is controllable from $x_{0}$ if $\mathcal{A}\left(x_{0}\right)=M$. In addition, it is controllable if it is controllable from any element of $M$. Let $t>0$, for technical reasons sometimes it is necessary to consider the set $\mathcal{A}\left(x_{0}, t\right)$, i.e., the reachable set from $x_{0}$ through the controls $u \in \mathcal{U}$ up to the time $t$. In addition, the set $\mathcal{A}^{*}\left(x_{0}\right)$ which are the elements that can be carried to the state $x_{0}$ in positive time through the system.

Like the tumor treatment problem, similar questions can be asked in the real world for many situations. From a practical point of view, given a manifold $M$, the drift $f$ to be controlled and the control vectors $g^{1}, \ldots, g^{m}$, the admissible class of control $\mathcal{U}$ must be properly chosen, according to any real situation.

To establish the Equivalence Theorem, we need to introduce the notion of Lie brackets between vector fields, given by the formula,

$$
[X, Y]=X . Y-Y . X, \text { where } X . Y=\frac{\partial}{\partial X} Y .
$$

The Lie algebra $\operatorname{Span}_{\mathcal{L} A}\left\{f, g^{1}, \ldots, g^{m}\right\}$ denotes the small vector space generated by $f, g^{1}, \ldots, g^{m}$ and closed by the bracket $[$,$] .$ 
Theorem 1. (Jouan Equivalence Theorem, [14]) If for $\Sigma_{M}$

$$
\operatorname{dim}\left(\operatorname{Span}_{\mathcal{L} A}\left\{f, g^{1}, \ldots, g^{m}\right\}\right)<\infty
$$

then, there exits a Lie group $G$ such that $\Sigma_{M}$ is equivalent to $\Sigma_{G}$ or, it is equivalent to $\Sigma_{G / H}$, where $\mathrm{G} / \mathrm{H}$ is a homogeneous space.

Equivalent systems share the same topological, dynamic and algebraic properties. Therefore, it is possible to get information of any arbitrary system $\Sigma_{M}$ which satisfy the Jouan condition through a linear system $\Sigma_{G}$ or via a homogeneous system $\Sigma_{G / H}$. Here, $H$ is a closed subgroup of $G$. This is one of the main reasons it is necessary to classify $\Sigma_{G}$ for different classes of groups: compact, non-compact, Abelian, nilpotent, solvable, simple, semi-simple, and the direct and semi-direct product between them. Since 1999, a group of mathematician had been working in the structure of $\Sigma_{G}$. Results about controllability, and the existence, uniqueness, and topological properties of control sets, (maximal regions of controllability), were established for several classes of groups. We refer the readers to the following [14-25].

As mentioned before, the main goal of this review is to invite the community to search for real world applications of $\Sigma_{G}$. However, there are also several theoretical open problems to be challenging. For instance, we mention that except for the Euclidean case, any other classical optimal issue for $\Sigma_{G}$, such as the quadratic one, is an open problem for $\Sigma_{G}$.

According to the aim of this review article, we concentrate on matrix groups. Precisely, for a group $G$ of dimension 2 and 3, we describe the main ingredients to build $\Sigma_{G}$ by showing a basis of the Lie algebra $\mathfrak{g}$ of $G$, the face of $\mathfrak{g}$-derivations and all possible associated linear vector fields. We also mention the existent controllability results and the Hamiltonian equations to apply the Pontryagin Maximum Principle in these cases.

For a general view of the theory of control systems we refer the readers to [1,2,26-32]. For the Lie theory, we mention [33-36]. For the connection with Sub-Riemannian, almostRiemmanian geometry we suggest the references [37].

This article is organized as follows. Section 2 introduces the algebraic structures to define $\Sigma_{G}$. Section 3 contains an explicit description of linear control systems and their main properties on groups of dimensions 2 and 3. In Section 4, we establish the Pontryagin Maximum Principle for $\Sigma_{G}$. Finally, in Section 5 we end with several examples. We include a classical optimal problem on the Euclidean plane, several examples on the 2-dimensional solvable group, and a time-optimal theoretical problems on 3-dimensional groups.

\section{Matrix Groups Dynamics and Systems}

In the sequel, $\mathfrak{g l}(n, \mathbb{R})$ will denote the vector space of the $n$ by $n$ matrices with real coefficients. The open set $G L(n, \mathbb{R})=\left[\operatorname{det}(A)^{-1}(0)\right]^{c} \subset \mathfrak{g l}(n, \mathbb{R})$, is the group of invertible matrices. In fact, $\mathfrak{g l}(n, \mathbb{R})$ is canonically isomorphic to $\mathbb{R}^{n^{2}}$, the determinant map $\operatorname{det}: \mathfrak{g l}(n, \mathbb{R}) \rightarrow \mathbb{R}$ is continuous, and $\operatorname{det}(A)^{-1}(0)$ is closed. We also denote by $G L^{+}(n, \mathbb{R})$ the connected component of $G L(n, \mathbb{R})$ which contains the identity element $I d=e$. In particular, for any $A \in G L^{+}(n, \mathbb{R})$, $\operatorname{det}(A)>0$. It turns out that the topology and the differentiable structure of $G L^{+}(n, \mathbb{R})$ are the induced ones, coming directly from the Euclidean space $\mathbb{R}^{n^{2}}$. Here, we just consider closed or path connected subgroups $G$ of $G L^{+}(n, \mathbb{R})$. Thus, $G$ is a matrix Lie group. In particular, it is possible to define appropriate control systems whenever the dynamic determining the system is well defined on $G$. The notion of a linear control system on a connected Lie group $G$ depends on two different classes of dynamics: linear and invariant vector fields. For that, we need to introduce the notion of the Lie algebra $\mathfrak{g}$ of $G$, which is isomorphic to the tangent space $T_{\mathcal{e}} G$ of $G$ at the identity element $e$. The tangent space $T_{g} G$ of $G$ at $g$ reads

$$
T_{g} G=\left\{Y_{g}: \exists \gamma:(-\varepsilon, \varepsilon) \stackrel{\text { continuous }}{\longrightarrow} G, \gamma(0)=g \text { and } \exists \dot{\gamma}(0)=Y_{g}\right\} \text {, }
$$


and $T G=\cup_{g \in G} T_{g} G$ denotes the tangent bundle.

A vector field $P$ on $G$ is defined by the selection of $P_{g} \in T_{g} G$ for any $g \in G$. Since we consider just subgroups $G$ of $G L^{+}(n, \mathbb{R})$, it follows that

$$
T_{g} G \subset T_{g} G L^{+}(n, \mathbb{R})=g+T_{e} G L^{+}(n, \mathbb{R})=g+\mathfrak{g l}(n, \mathbb{R}) .
$$

For matrices, the bracket $[X, Y]=X Y-Y X$, is the commutator.

By definition, the drift $\mathcal{X}$ is a linear vector field if its flows $\left\{\mathcal{X}_{t}: t \in \mathbb{R}\right\}$ is a 1-parameter group of $\operatorname{Aut}(G)$, the group of $G$-automorphisms. Moreover, $\mathcal{X}$ induced a derivation,

$$
\mathcal{D}(\mathcal{X})=\mathcal{D}: \mathfrak{g} \longrightarrow \mathfrak{g}: \mathcal{D}[X, Y]=[\mathcal{D} X, Y]+[X, \mathcal{D} Y], X, Y \in \mathfrak{g},
$$

i.e., a linear transformation which respects the Leibnitz rule for the Lie bracket. Furthermore, $\mathcal{X}$ is computed through the following identities,

$$
\mathcal{X}_{g}=\left(\frac{d}{d t}\right)_{t=0} \mathcal{X}_{t}(g), \mathcal{X}_{t}(\exp Y)=e^{t \mathcal{D}} Y, \text { and } \mathcal{X}_{t}(g h)=\mathcal{X}_{t}(g) \mathcal{X}_{t}(h)
$$

In fact, since we just consider connected groups, any element $g \in G$ is a finite product of exponential members of $\mathfrak{g}$.

A special situation happens when the Lie algebra $\mathfrak{g}$ is semi-simple, i.e., when the solvable radical $r(\mathfrak{g})$ of $\mathfrak{g}$ is trivial. Here, $r(\mathfrak{g})$ is defined as the biggest solvable subalgebra of $\mathfrak{g}$. In this case, any derivation $\mathcal{D}$ is inner. It turns out that there exists an invariant vector field $Y=Y(\mathcal{D})$, such that $\mathcal{D}=[, Y]$. Therefore, $\mathcal{D}$ is easily computed by

$$
\mathcal{D}=-a d(Y) \Longrightarrow \mathcal{X}_{g}=g Y-Y g, g \in G \text {. }
$$

Denote by $T^{n}=S^{1} \times \ldots \times S^{1},\left(n\right.$-times) the Torus. Therefore, $\operatorname{Aut}\left(T^{n}\right)=S L(n, Z)$ is the discrete group of determinant 1 matrices of order $n$ with integer coefficients. It turns out that any linear vector field $\mathcal{X}$ on $T^{n}$ is trivial, i.e., $\mathcal{X}_{g}=0$. In fact, $\left\{\mathcal{X}_{t}: t \in \mathbb{R}\right\}$ should be discrete. Because of that, we do not consider $T^{n}$ in this analysis.

An invariant vector field is determined by G-translations. Precisely, to define a left invariant vector field $Y$ on $G$ we just need to determine a tangent vector at the identity element $Y=Y_{e}$. Thus, for any $g \in G$ its value $Y_{g} \in T_{g} G$ is determined by the derivative at $e$ of the corresponding left translation $L_{g}$. Precisely,

$$
Y_{g}=\left(d L_{g}\right)_{e}\left(Y_{e}\right), \text { where }\left(d L_{g}\right)_{e}: T_{e} G \rightarrow T_{g} G
$$

Since we work with matrices, it turns out that $Y_{g}$ is obtained by the derivative of the curve $\gamma(t)=g \exp (t Y)$.Therefore, $Y_{g}=g Y$, with $Y_{e}=Y$. Thus, $\mathfrak{g}$ is isomorphic to the tangent space $T_{e} G$. Right invariant vector fields are defined in a similar way. The vector space $\mathfrak{g}$ of the left invariant vector fields of $G$, is a Lie algebra $[35,36]$. The skew-symmetric bilinear map [,] $: \mathfrak{g} \times \mathfrak{g} \rightarrow \mathfrak{g}$, satisfy

1. $[P, Q]=-[Q, P]$ skew-symmetric, and the Jacobi identity

2. $[X,[Y, Z]]+[Z,[X, Y]]+[Y,[Z, X]]=0$, for any $X, Y, Z \in \mathfrak{g}$.

Recall that the Lie algebra $\mathfrak{g}$ is said to be:

Abelian, if for any $X, Y \in \mathfrak{g},[X, Y]=0$.

Nilpotent, if $\exists k \geq 1: \mathbf{a d}^{1}=[\mathfrak{g}, \mathfrak{g}] \supset \ldots \supset \mathbf{a d}^{k+1}=\left[\mathbf{a d}^{k}, \mathfrak{g}\right]=0$.

Solvable, if $\exists k \geq 1: \mathbf{a d}^{1} \supset \ldots \supset \operatorname{ad}^{(k)}=\left[\operatorname{ad}^{(k-1)}, \mathbf{a d}^{(k-1)}\right]=0$.

Semisimple, if the largest solvable subalgebra $\mathfrak{r}(\mathfrak{g})$ of $\mathfrak{g}$ is trivial.

Finite semi-simple center if any semi-simple subalgebra has a trivial center.

Finally, the group $G$ is said to be Abelian, nilpotent, solvable, semi-simple, and finite semi-simple center, if $\mathfrak{g}$ satisfies the corresponding property. 


\subsection{The Notion of Linear Control System on $G$}

Let $G$ be a connected Lie group with Lie algebra $\mathfrak{g}$. By definition, see [20,25], a linear control systems $\Sigma_{G}$ is determined by the differential equations

$$
\Sigma_{G}: \dot{g(t)}=\mathcal{X}(g(t))+\sum_{j=1}^{m} u_{j}(t) Y^{j}(g(t)), g(t) \in G, u \in \mathcal{U},
$$

parametrized by $u \in \mathcal{U}$. Here, $\mathcal{X}$ is a linear vector field which means that its flows $\left\{\mathcal{X}_{t}: t \in \mathbb{R}\right\} \subset \operatorname{Aut}(G)$ is a 1-parameter group of $G$-automorphisms. In addition, for any $j=1, \ldots, m$, the control vector $Y^{j} \in \mathfrak{g}$ is a left invariant vector field. We consider a large class of admissible control functions

$$
\mathcal{U}=\left\{u:\left[0, T_{u}\right] \longrightarrow \Omega \subset \mathbb{R}^{m}: \mathrm{u} \text { is locally integrable }\right\},
$$

where $\Omega$ is a closed and convex subset with $0 \in \operatorname{int}(\Omega)$. Just observe that $\mathcal{U}$ contains the constant, continuous and also differentiable functions.

Because of that, given any initial condition $g \in G$ and each control $u \in \mathcal{U}$ there exist a solution $\varphi(g, u, t)$ of $\Sigma_{G}$. Precisely,

$$
\varphi(g, u, t)=\mathcal{X}_{t}(g) \varphi(e, u, t),
$$

where $\varphi(e, u, t)$ is the solution of the system with the same control $u$ through the identity element $e$ [20]. We denote the reachable set from $e$ by

$$
\begin{gathered}
\mathcal{A}=\{\varphi(e, u, t): u \in \mathcal{U}\}, \text { and the set, } \\
\mathcal{A}^{*}=\{g \in G: \exists u \in \mathcal{U}, \text { and } t>0, \text { with } \varphi(g, u, t)=e\},
\end{gathered}
$$

of state of the group that can be transferred to the identity in a positive time.

We call the system unbounded if $\Omega=\mathbb{R}^{m}$, and bounded if $\Omega$ is compact. From now $\mathcal{U}_{\mathbb{R}^{m}}$ will denote the unbounded case, $\mathcal{U}_{\Omega}$ the bounded one and $\mathcal{U}$ when both condition are possible.

Therefore, the notion of $\Sigma_{G}$ is a natural extension of the classical linear control system $\Sigma_{\mathbb{R}^{n}}$ on the Abelian group $\mathbb{R}^{n}$

Recall that the system satisfy the Lie algebra rank condition ( $L A R C)$, if

$$
\operatorname{Span}_{\mathcal{L A}}\left\{\mathcal{X}, Y^{1}, \ldots, Y^{m}\right\}=\mathfrak{g}
$$

Furthermore, the system satisfies the ad-rank condition $a d R C$ if

$$
\operatorname{Span}\left\{\mathcal{D}^{k}\left(Y^{j}\right): j=1, \ldots, m, k \geq 0\right\}=\mathfrak{g} .
$$

We denote by $\Delta$ the Lie algebra generated by the control vectors, i.e.,

$$
\Delta=\operatorname{Span}_{L A}\left\{Y^{1}, \ldots, Y^{m}\right\} .
$$

Finally, we introduce the notion of a control set, which means a region where controllability holds in its interior. Let $\Sigma_{G}$ be a linear control system. A subset $\mathcal{C}$ is said to be a control set if

1. for any $g \in G, \exists u$ such that $\varphi(g, u, t) \in \mathcal{C}, t \geq 0$

2. $\mathcal{C} \subset \operatorname{cl}(\mathcal{A}(g)$, for any $g \in \mathcal{C}$

3. $\quad \mathcal{C}$ is maximal with respect 1 and 2 .

Furthermore, the control set $\mathcal{C}$ is said to be positive invariant if,

$$
g \in \mathcal{C} \Rightarrow \varphi(g, u, t) \in \mathcal{C}, \text { for any } u \in \mathcal{U}, t>0 \text {, }
$$

Here, $c l$ denotes the topological closure. 


\subsection{The Classical Linear Control System on $\mathbb{R}^{n}$}

In the Euclidean Abelian group

$$
\mathbb{R}^{n}=\left\{x=\left(x_{1}, x_{2}, \ldots, x_{n}\right): x_{i} \in \mathbb{R}, i=1, \ldots, n\right\}
$$

the classical linear control system is as follows

$$
\Sigma_{\mathbb{R}^{n}}: \dot{x}(t)=A x(t)+B u, u \in \mathcal{U} .
$$

In this case, $\mathcal{D}$ corresponds to the matrix $A$ of order $n$, which flows $\left\{e^{t A}: t \in \mathbb{R}\right\} \subset$ $\operatorname{Aut}\left(\mathbb{R}^{n}\right)$ is computed from the exponential map. On the other hand, $B u \in \mathbb{R}^{n}$ can be written as $\sum_{j=1}^{m} u_{j} b^{j}$, where $b^{1}, \ldots, b^{m}$, are the column vectors of the $n$ by $m$ matrix $B$. Since, any vector $b \in \mathbb{R}^{n}$ induces by translation an invariant vector field on $\mathbb{R}^{n}$, it is now clear that $\Sigma_{G}$ is a perfect extension of $\Sigma_{\mathbb{R}^{n}}$.

It is well known that the solution with initial condition and control $u$ is given by,

$$
\varphi\left(x_{0}, u, t\right)=e^{t A} x_{0}+\int_{0}^{t} e^{-\tau A} B u(\tau) d \tau .
$$

\subsection{The $\mathcal{D}$-Decomposition of $\mathfrak{g}$}

The dynamic behavior of $\Sigma_{G}$ strongly depends on the spectrum of $\mathcal{D}$ associated with $\mathcal{X}$. We consider the Lie algebra decomposition of $\mathfrak{g}$ induced by $\mathcal{D}$. For any $\alpha \in \operatorname{Spec}(\mathcal{D})$, the $\mathcal{D}$-generalized eigenspaces reads

$$
\mathfrak{g}_{\alpha}=\left\{Y \in \mathfrak{g}:(\mathcal{D}-\alpha I d)^{n} Y=0, \text { for some } n \geq 1 .\right.
$$

It turns out that $\left[\mathfrak{g}_{\alpha}, \mathfrak{g}_{\beta}\right] \subset \mathfrak{g}_{\alpha+\beta}$ if $\alpha+\beta \in \operatorname{Spec}(\mathcal{D})$ and 0 otherwise. In addition, $\mathfrak{g}$ decomposes as $\mathfrak{g}=\mathfrak{g}^{+} \oplus \mathfrak{g}^{0} \oplus \mathfrak{g}^{-}$, where

$$
\mathfrak{g}^{+}=\bigoplus_{\operatorname{Re}(\alpha)>0} \mathfrak{g}_{\alpha}, \mathfrak{g}^{0}=\bigoplus_{\operatorname{Re}(\alpha)=0}^{\bigoplus} \mathfrak{g}_{\alpha} \text { and } \mathfrak{g}^{-}=\underset{\operatorname{Re}(\alpha)<0}{\bigoplus} \mathfrak{g}_{\alpha} .
$$

$\mathfrak{g}^{+}, \mathfrak{g}^{0}, \mathfrak{g}^{-}$are Lie subalgebras and $\mathfrak{g}^{+}, \mathfrak{g}^{-}$are nilpotent [22,35].

We finish this section bt mentioning some differences between $\Sigma_{G}$ and the well-known class of invariant control system $I n v_{G}$ on $G[38,39]$. For $I n v_{G}$ the drift is also an element of the Lie algebra $\mathfrak{g}$. One of the main differences comes from the fact that the reachable set from the identity is a semigroup for $I n v_{G}$ but not for $\Sigma_{G}$. This difference has important consequences. For example, the controllability property of $I n v_{G}$ turns into a local property, which is no the case for $\Sigma_{G}$ [19]. On the other hand, the Hamiltonian lifting of the linear vector field is more complicated because the invariant field does not depend on the state.

\section{Linear Control Systems and Controllability}

For a group $G$ of dimension 2 and 3 , in the sequel, we describe the main ingredients to build $\Sigma_{G}$. We show a basis of the Lie algebra $\mathfrak{g}$ of $G$, the form of all $\mathfrak{g}$-derivations, and the associated linear vector fields. We also mention some normal form of $\Sigma_{G}$ associated with the $L A R C$ property, which we always assume, without loss of generality. Finally, we characterize the controllability property of each system, (A few words about assuming $L A R C$. For a regular (constant dimension) and involutive (closed by Lie brackets) distribution $\Psi$ generated by a family of vector fields $\Gamma$ on a manifold $M$, the Sussmann Orbit Theorem implies that the integral manifold $M(\Psi)$ of $\Psi$, a submanifold of $M$ is built by the concatenation of the integral curves of the vector fields in $\Gamma$, in positive and negative time. In particular, if $M(\Psi) \varsubsetneqq M$ we just consider $\Gamma$ on $M(\Psi)$. Therefore, by assuming that $\Sigma_{G}$ satisfy the $L A R C$ property, it turns out that $G(\Psi)=G$, when

$$
\left.\Psi=\operatorname{Span}_{\mathcal{L} A}\left\{\mathcal{X}, Y^{1}, \ldots, Y^{m}\right\} .\right)
$$




\subsection{The Dimension 2}

In dimension two, there are just two Lie algebras: the Abelian and solvable.

\subsubsection{The Abelian Structure}

The Lie algebra $\mathbb{R}^{2}$ is generated by the basis $X=e_{1}, Y=e_{2}$, with $[X, Y]=0$. The associated group is $\mathbb{R}^{2}$ with the Abelian structure

$$
\left(x_{1}, y_{1}\right)+\left(x_{2}, y_{2}\right)=\left(x_{1}+x_{2}, y_{1}+y_{2}\right)
$$

induced by its Lie algebra. The Left invariant vector fields are given by

$$
X_{g}=\frac{\partial}{\partial x}, Y_{g}=\frac{\partial}{\partial y}, \text { where } g=x e_{1}+y e_{2}
$$

Any matrix $\mathcal{D}=A=\left(\begin{array}{ll}a_{11} & a_{12} \\ a_{21} & a_{22}\end{array}\right), a_{i, j} \in \mathbb{R}$ where $i, j=1,2$, is a derivation, determining the linear vector field $\dot{x}(t)=A x(t)$. Therefore, any classical linear control system on the Abelian group has the face

$$
\Sigma_{\mathbb{R}^{2}}:\left(\begin{array}{c}
\dot{x}(t) \\
\dot{y}(t)
\end{array}\right)=A\left(\begin{array}{c}
x(t) \\
y(t)
\end{array}\right)+\left(\begin{array}{c}
b_{1} \\
b_{2}
\end{array}\right) u, u \in \mathcal{U}_{\mathbb{R}} \text {, or } u \in \mathcal{U}_{\Omega} .
$$

In reference [27] it is possible to find the controllability results for the unbounded and bounded admissible control functions, see also [29]. If $\mathcal{U}=\mathcal{U}_{\mathbb{R}^{2}}$, the LARC property is equivalent to the controllability property, which is equivalent to the Kalman rank condition. Precisely,

Theorem 2. Let $\Sigma_{\mathbb{R}^{2}}$ be a linear control system with $\mathcal{U}=\mathcal{U}_{\mathbb{R}^{2}}$. Then,

$$
\Sigma_{\mathbb{R}^{2}} \text { is controllable } \Leftrightarrow \operatorname{rank}(b A b)=2, b=\left(b_{1} b_{2}\right)^{T} .
$$

Furthermore, consider $u_{*}<0<u^{*}$ and $\Omega=\left[u_{*}, u^{*}\right]$. If $\mathcal{U}=\mathcal{U}_{\Omega}$, it holds

1. $\Sigma_{\mathbb{R}^{2}}$ is controllable $\Leftrightarrow$ Kalman rank condition and $\operatorname{Spec}_{L y}(\mathcal{D})=\{0\}$.

2. Around the identity element 0 , there exists an unique control set with non-empty interior, given by

$$
\mathcal{C}=\operatorname{cl}(\mathcal{A}) \cap \mathcal{A}^{*}
$$

\subsubsection{The Solvable Structure}

Here, we follow the reference [24]. The Lie algebra $\mathfrak{a} f f_{+}(\mathbb{R})$ is realized as the vector space of the real matrices of order 2 generated by $X=\left(\begin{array}{ll}1 & 0 \\ 0 & 0\end{array}\right)$,

$Y=\left(\begin{array}{ll}0 & 1 \\ 0 & 0\end{array}\right)$, with $[X, Y]=Y$. The simply connected Lie group reads as

$$
G=\left\{g=\left(\begin{array}{cc}
x & y \\
0 & 1
\end{array}\right): x>0, y \in \mathbb{R}\right\} \cong A f f_{+}(\mathbb{R})=\mathbb{R}_{+} \times \mathbb{R},
$$

with the solvable algebraic structure

$$
\left(x_{1}, y_{1}\right) \times\left(x_{2}, y_{2}\right)=\left(x_{1} x_{2}, x_{1} y_{2}+x_{1}\right)
$$

The left invariant vector fields are given by $X_{g}=g X=x \frac{\partial}{\partial x}$ and $Y_{g}=g Y=x \frac{\partial}{\partial y}$. Any derivation 


$$
\mathcal{D}=\left(\begin{array}{ll}
0 & 0 \\
a & b
\end{array}\right), a, b \in \mathbb{R}
$$

is inner, and induces the linear vector field

$$
\mathcal{X}_{g}=\left(\begin{array}{cc}
0 & a(x-1)+b y \\
0 & 0
\end{array}\right), a, b \in \mathbb{R} .
$$

Next, we follow reference Jouan. The linear system $\Sigma_{G}$

$$
\dot{g}=\mathcal{X}_{g}+u g(c X+d Y)
$$

satisfies $L A R C$ if and only if

$$
\Sigma_{G}:\left\{\begin{array}{l}
\dot{x}=u c x, c \neq 0 \\
\dot{y}=x-1+b y
\end{array}, u \in \mathcal{U} .\right.
$$

In this case, the linear system $\Sigma_{G}$ is said to be in normal form.

Theorem 3 ([24]). Assume, $\Sigma_{G}$ is in normal form, therefore

$$
\Sigma_{G} \text { is controllable } \Leftrightarrow b=0 .
$$

In [15], the authors compute the control set of any linear control system $\Sigma_{G}$ on $G$, with and without non-empty interior. There are six classes. Here, we just show three.

1. Let us consider

$$
\Sigma_{G}:\left\{\begin{array}{l}
\dot{x}=u x \\
\dot{y}=b y
\end{array}, \text { where } u \in \Omega=\left[u_{*}, u^{*}\right],\right.
$$

whose solutions starting at $(x, y) \in G$ are given by concatenations of the flows

$$
\varphi(t,(x, y), u)=\left(\mathrm{e}^{t u} x, \mathrm{e}^{t b} y\right), \quad t \in \mathbb{R} .
$$

As a matter of fact, the only control set is given by $\mathcal{C}=\mathbb{R}_{+} \times\{0\}$.

2. Let us consider the linear system

$$
\Sigma_{G}:\left\{\begin{array}{l}
\dot{x}=0 \\
\dot{y}=a(x-1)+u x
\end{array}, \text { where } u \in \Omega=\left[u_{*}, u^{*}\right]\right.
$$

whose solution with initial condition $(x, y)$ and control $u$ is given by

$$
\varphi((x, y), u, t)=(x,(a(x-1)+u x) t+y), t \in \mathbb{R} .
$$

Here, there are an infinity number of control sets. In fact, for any $x \in \mathbb{R}$

$$
\mathcal{C}_{x}=\{x\} \times \mathbb{R} \text {, when } x \in(1-\varepsilon, 1+\varepsilon) \text {, and any } y \in \mathbb{R}
$$

for an existent positive number $\varepsilon$.

3. Consider the linear system

$$
\Sigma_{G}:\left\{\begin{array}{l}
\dot{x}=u x \\
\dot{y}=b y+u x
\end{array}, \text { where }, b<0, u \in \Omega=\left[u_{*}, u^{*}\right]\right.
$$

In this case it is possible to prove that the only one control set with non-empty interior is given by

$$
\mathcal{C}=\operatorname{cl} \mathcal{A}(x, y) \text { for any }(x, y) \in \mathcal{C}
$$




\subsection{The Dimension 3}

In this section, we describe the ingredients to build a linear control system on any 3-dimensional matrix Lie group. In particular, in the Abelian, nilpotent, solvable, finite center, semi-simple compact, and non-compact case.

\subsubsection{The Abelian Structure}

Here, $\mathbb{R}^{3}$ is generated by the basis $X=e_{1}, Y=e_{2}, Z=e_{3}$. In addition, all the brackets are null. The associated group is also $\mathbb{R}^{3}$ with the Abelian structure

$$
\left(x_{1}, y_{1}, z_{1}\right)+\left(x_{2}, y_{2}, z_{2}\right)=\left(x_{1}+x_{2}, y_{1}+y_{2}, z_{1}+z_{2}\right),
$$

induced by its Lie algebra. The Left invariant vector fields are given by

$$
X_{g}=\frac{\partial}{\partial x}, Y_{g}=\frac{\partial}{\partial y}, Z_{g}=\frac{\partial}{\partial z} \text { where } g=x e_{1}+y e_{2}+z e_{3} .
$$

Any matrix $\mathcal{D}=A=\left(a_{i, j}\right), a_{i, j} \in \mathbb{R} i, j=1,2,3$, is a derivation, determining the linear vector field $\dot{x}(t)=A x(t)$. Therefore, any classical linear control system on the Abelian group has the face

$$
\Sigma_{\mathbb{R}^{3}}: \dot{x}(t)=A x(t)+B u(t), u \in \mathcal{U} .
$$

Here, the matrix $B=\left(b_{i, j}\right)$, with the possibilities: $i=1,2,3 ; j=1$, and $u_{1} \in \mathcal{U}$, or, $i=1,2,3 ; j=1,2$ and $u_{1} \in \mathcal{U}, u_{2} \in \mathcal{U}$.

In reference [27] it is possible to find the controllability results for the unbounded and bounded admissible control functions, see also [29]. If $\mathcal{U}=\mathcal{U}_{\mathbb{R}^{3}}$, the $L A R C$ property is equivalent to the controllability property which, is also equivalent to the Kalman rank condition. Precisely,

Theorem 4. Let $\Sigma_{\mathbb{R}^{3}}$ be a linear control system with $\mathcal{U}=\mathcal{U}_{\mathbb{R}^{3}}$. Then,

$$
\Sigma_{\mathbb{R}^{3}} \text { is controllable } \Leftrightarrow \operatorname{rank}\left(B A B A^{2} B\right)=3 .
$$

Furthermore, consider $u_{*}<0<u^{*}$ and $\Omega=\left[u_{*}, u^{*}\right]$. If $\mathcal{U}=\mathcal{U}_{\Omega}$, it holds

1. $\quad \Sigma_{\mathbb{R}^{3}}$ is controllable $\Leftrightarrow$ Kalman rank condition and $\operatorname{Spec}_{L y}(\mathcal{D})=\{0\}$.

2. Around the identity element 0 , there exists an unique control set with non-empty interior, given by

$$
\mathcal{C}=\operatorname{cl}(\mathcal{A}) \cap \mathcal{A}^{*}
$$

In what follows, we denote by $M(i, j)$, the matrix of order three with all coefficients 0 , except 1 at the position $(i, j)$.

\subsubsection{The Nilpotent Structure}

The Heisenberg Lie algebra is the only nilpotent Lie algebra of dimension three. It is generated by $X=M(1,2), Y=M(2,3)$, and $Z=M(1,3)$. The simply connected Heisenberg Lie group reads as

$$
G=\left\{\left(\begin{array}{ccc}
1 & x & z \\
0 & 1 & y \\
0 & 0 & 1
\end{array}\right): x, y, z \in \mathbb{R}\right\} \longleftrightarrow(x, y, z) \in \mathbb{R}^{3} .
$$

The left invariant vector fields and its brackets are given by

$$
X_{g}=\frac{\partial}{\partial x}, Y_{g}=\frac{\partial}{\partial y}+x \frac{\partial}{\partial z}, Z_{g}=\frac{\partial}{\partial z} ;[X, Y]=Z,[X, Z]=[Y, Z]=0 .
$$


An arbitrary derivation of the Heisenberg has the form,

$$
\mathcal{D}=\left(\begin{array}{ccc}
a & b & 0 \\
c & d & 0 \\
e & f & a+d
\end{array}\right): a, b, c, d, e, f \in \mathbb{R}
$$

and induces the general linear vector field on $G$,

$$
\mathcal{X}(g)=(a x+b y) \frac{\partial}{\partial x}+(c x+d y) \frac{\partial}{\partial y}+\left(e x+f y+(a+d) z+\frac{1}{2} c x^{2}+\frac{1}{2} b y^{2}\right) \frac{\partial}{\partial z} .
$$

Next, we follow reference [24]. The authors consider two different situations: one and two control vectors. On the Heisenberg Lie group, $\mathcal{U}=\mathcal{U}_{\mathbb{R}^{m}}$.

The single control vector

Any linear control system on the Heisenberg group satisfy the LARC property if and only if it is written in normal form,

$$
\Sigma_{G}: \dot{g}=\mathcal{X}_{g}+u X_{g}, u \in \mathcal{U},
$$

where $\mathcal{X}$ comes from the derivation $\left(\begin{array}{lll}0 & b & 0 \\ 1 & d & 0 \\ 0 & f & d\end{array}\right)$, and $X=M(1,2)$.

Theorem 5 ([24]). If $\Sigma_{G}$ is in normal form, then

$$
\dot{g}=\mathcal{X}_{g}+u X_{g} \text { is controllable } \Longleftrightarrow b<-\frac{d^{2}}{4} \Leftrightarrow d=0, f \neq 0 .
$$

It is possible to obtain algebraic conditions for a 1-input linear system on the Heisenberg group when the system is not written in normal form, Jouan.

The two control vectors

Any linear control system which satisfy $L A R C$ reads as follows,

$$
\Sigma_{G}: \dot{g}=\mathcal{X}_{g}+u_{1} P_{g}+u_{2} Q_{g}, u \in \mathcal{U}
$$

where $P, Q \in \mathfrak{g}$ are linear independent invariant vector fields on $G$.

Theorem 6 ([24]). Let $\Sigma_{\mathbb{G}}$ be a linear control system with two control vectors and $\mathcal{U}=\mathcal{U}_{\mathbb{R}^{3}}$. Then,

$$
\Sigma_{G} \text { is controllable } \Leftrightarrow \Sigma_{G} \text { satisfy LARC. }
$$

\subsubsection{The Solvable Structure}

According to Onishchik and Vinberg [34], there are five nonisomorphic, nonnilpotent solvable Lie algebras of dimension three. On the other hand, for these five classes of Lie algebras, the authors in [16], describe general formulas through the semi-direct product to compute the simply connected Lie groups associated with the Lie algebras, the derivations and its associated linear vector fields in any case. Furthermore, they characterize the controllability property on any linear control system which satisfies the LARC property for one and two control vectors. We also inform the readers that there is no information about control sets.

Let us consider the canonical basis,

$$
\mathfrak{g}=\operatorname{Span}\{X=(1,0,0), Y=(0,1,0), Z=(0,0,1)\}
$$

The classification goes through the notion of the semi-direct product between the Abelian Lie algebras $\mathbb{R}$ and $\mathbb{R}^{2}$. Precisely,

$$
\mathfrak{g}_{\theta}=\mathbb{R} \otimes_{\theta} \mathbb{R}^{2}:\left[\left(z_{1}, v_{1}\right),\left(z_{2}, v_{2}\right)\right]=\left(0, z_{1} \theta v_{2}-z_{2} \theta v_{1}\right) \in \mathfrak{g} .
$$


Up to automorphisms, these solvable Lie algebras depend on a matrix $\theta$.

The Lie algebra $\mathfrak{r}_{2}: \theta=\left(\begin{array}{ll}0 & 0 \\ 0 & 1\end{array}\right),[X, Z]=Z,[X, Y]=0$.

The Lie algebra $\mathfrak{r}_{3}: \theta=\left(\begin{array}{ll}1 & 1 \\ 0 & 1\end{array}\right),[X, Y]=Y,[X, Z]=Y+Z$.

The Lie algebra $\mathfrak{r}_{3, \lambda}: \theta=\left(\begin{array}{cc}\lambda & -1 \\ 1 & \lambda\end{array}\right), \lambda \in \mathbb{R}, \lambda \neq 0,[X, Y]=Z$,

$[X, Z]=-Y$.

The Lie algebra $\mathfrak{r}_{3, \lambda}^{*}: \theta=\left(\begin{array}{cc}\lambda & -1 \\ 1 & \lambda\end{array}\right), \lambda \in \mathbb{R}, \lambda \neq 0,[X, Y]=\lambda Y$,

$$
[X, Z]=-Y+\lambda Z \text {. }
$$

The Lie algebra $\mathfrak{e}: \theta=\left(\begin{array}{cc}0 & -1 \\ 1 & 0\end{array}\right),[X, Y]=Z,[X, Z]=-Y$.

Let us show how it works this special product. By the own definition, for every solvable class, $[Y=(0,1,0), Z=(0,0,1)]=(0,0,0)$. For $\mathfrak{r}_{2}$, the bracket $[X, Z]$ is computed as follows

$$
[X, Z]=\left(0,\left(\begin{array}{ll}
0 & 0 \\
0 & 1
\end{array}\right)\left(\begin{array}{l}
0 \\
1
\end{array}\right)-\left(\begin{array}{l}
0 \\
0
\end{array}\right)\right)=Z \text {. }
$$

The solvable Lie groups

The simply connected Lie groups $R_{2}, R_{3}, R_{3, \lambda}, R_{3, \lambda}^{*}$ and $E$ with Lie algebras $\mathfrak{r}_{2}, \mathfrak{r}_{3}, \mathfrak{r}_{3, \lambda}, \mathfrak{r}_{3, \lambda}^{*}$ and $\mathfrak{e}$ respectively, are computed by the semi-direct product $G_{\theta}=\mathbb{R} \otimes_{\rho} \mathbb{R}^{2}$ through the representation $\rho$ and the formula

$$
\rho_{\theta}(t)=e^{t \theta}, \text { where } e^{t \theta}=\sum_{n=0}^{\infty} \frac{t^{n}}{n !} \theta^{n}, \theta^{0}=I d, t \in \mathbb{R} .
$$

Furthermore, any connected Lie group $G$ which solvable Lie algebra is determined by the matrix $\theta$, is given by a homogeneous space $G_{\theta} / P$, where $P$ is a discrete subgroup of $G_{\theta}$. For more details, see [16].

\section{Linear and left invariant vector fields}

Next, we show the face of the dynamic involved in $\Sigma_{G}$ on any three dimensional solvable non-nilpotent simply connected Lie group $G$.

$$
\begin{array}{ccc}
\text { Algebra, Group } & \text { Left invariant vector fields } & \text { Linear vector fields } \\
\mathfrak{g}_{\theta}=\mathbb{R} \otimes_{\theta} \mathbb{R}^{2} & Y=(a, w) \in \mathfrak{g}_{\theta} & \mathcal{X}(t, 0)=\left(0, \Lambda_{t} \xi\right) \\
G_{\theta}=\mathbb{R} \otimes_{\rho} \mathbb{R}^{2} & Y(t, v)=\left(a, \rho_{t} w\right) & \mathcal{X}(t, v)=\left(0, \mathcal{D}^{*} v+\Lambda_{t} \xi\right)
\end{array}
$$

where $\mathcal{D}^{*}$ is defined through the formula $\mathcal{D}(0, v)=\left(0, \mathcal{D}^{*} v\right)$, and

$$
\Lambda_{t}=\left\{\begin{array}{cc}
\left(\rho_{s}-1\right) \theta^{-1} & \operatorname{det}(\theta) \neq 0 \\
\left(\begin{array}{cc}
s & 0 \\
0 & e^{s}-1
\end{array}\right) & \operatorname{det}(\theta)=0 .
\end{array}\right\} .
$$

Any linear control system $\Sigma_{G}$ on a three-dimensional, solvable, connected, nonnilpotent Lie group $G$ that satisfies $L A R C$ is equivalent to one of the following linear systems:

1. The one control vector,

$$
\dot{g}=\mathcal{X}(g)+u Y^{1}(g), \text { where } Y_{1}=(1,0) .
$$

2. The two control vectors,

$$
\dot{g}=\mathcal{X}(g)+u_{1} Y^{1}(g)+u_{2} Y^{2}(g), \text { with } Y_{1}=(1,0), Y_{2}=(0, w), \text { some } w \in \mathbb{R}^{2}
$$


Next, we present a characterization of the controllability property in both cases.

The single control vector

As always, we assume the $L A R C$ property.

Theorem 7 ([16]). Let $\Sigma_{G}: \dot{g}=\mathcal{X}(g)+u Y^{1}(g), Y_{1}=(1,0)$. Then,

1. If $G=R_{2}: \Sigma_{G}$ is controllable $\Leftrightarrow \mathfrak{g}^{0} \simeq \mathfrak{a f f}(\mathbb{R})$

2. If $G=E_{2}$ or $R_{3}: \Sigma_{G}$ is controllable $\Leftrightarrow \mathfrak{g}=\mathfrak{g}^{0}$ and $\mathcal{D}^{*} \not \equiv 0$

3. If $G=R_{3, \lambda}: \Sigma_{G}$ is controllable $\Leftrightarrow \lambda=1$ and $\mathcal{D}^{*}$ has a pair of complex eigenvalues

4. If $G=R_{3, \lambda}^{\prime}: \Sigma_{G}$ is controllable.

The two control vectors

Let $\Sigma_{G}$ be a linear control system with two control vectors which satisfy the $L A R C$ property. For the controllability property it holds,

Theorem 8 ([16]). Let $\Sigma_{G}: \dot{g}=\mathcal{X}(g)+u_{1} Y^{1}(g)+u_{2} Y^{2}(g)$,

$Y_{1}=(1,0)$ and $Y_{2}=(0, w)$, for some $w \in \mathbb{R}^{2}$. Then,

Theorem 9. 1. If $G=R_{2}: \Sigma_{G}$ is controllable $\Leftrightarrow \operatorname{dim} \mathfrak{g}^{0}>1$ or $\operatorname{dim} \mathfrak{g}^{0}=1$ and $\Delta \simeq \mathfrak{a f f}(\mathbb{R})$

2. If $G=E_{2}$, or $R_{3, \lambda}^{\prime}: \Sigma_{G}$ is controllable

3. If $G=R_{3}: \Sigma_{G}$ is controllable $\Leftrightarrow \mathfrak{g}=\mathfrak{g}^{0}$

4. If $G=R_{3, \lambda}: \Sigma_{G}$ is controllable $\Leftrightarrow \operatorname{ker} \mathcal{D}^{*} \not \subset \Delta$ or $\mathcal{D}$ has a pair of complex eigenvalues.

\subsubsection{The Finite Semi-Simple Center Structure}

In [18] the authors introduce the notion of a finite semi-simple Lie algebra $\mathfrak{g}$, which means that any semi-simple subalgebra of $\mathfrak{g}$ has a finite center. In particular, any solvable Lie algebra has this property. Any semi-simple Lie algebra with a finite center such as the skew-symmetric algebra $\mathfrak{s o}(n, \mathbb{R})$ and $\mathfrak{s l}(n, \mathbb{R})$, the trace zero matrices of order $n$. In both cases the center $\mathfrak{z}$ is discrete: the identity $I d$, and $\pm I d$, respectively. In the sequel, we consider two topological different cases.

\subsubsection{The Compact Semi-Simple Structure}

The vector space $\mathfrak{g}=\operatorname{Span}\{X, Y, Z\}$ is the Lie algebra of skew-symmetric matrices of order three, generated by the basis

$$
X=M(1,2)-M(2,1), Y=M(2,3)-M(3,2), M(1,3)-M(3,1) .
$$

The brackets rules are $[X, Y]=Z,[X, Z]=-Y,[Y, Z]=X$.

Since $\mathfrak{g}$ is semi-simple, any derivation is inner. It turns out that $\mathcal{D}=-a d(U)$, for $U \in \mathfrak{s o}(3, \mathbb{R})$. Thus, the linear vector field associated to $\mathcal{D}$ reads

$$
\mathcal{X}_{g}=g U-U g, U \in \mathfrak{g} .
$$

The connected Lie group with Lie algebra $\mathfrak{s o}(3, \mathbb{R})$ is the orthogonal group with positive determinant $S O(3, \mathbb{R})$, i.e., it is the rotational group of $\mathbb{R}^{3}$.

Any linear control system on the compact group $S O(3, \mathbb{R})$, reads as

$$
\Sigma_{G}: \dot{g}=\mathcal{X}_{g}+u_{1} P_{g}+u_{2} Q_{g}, P, Q \in \mathfrak{g}, u_{1}, u_{2} \in \mathcal{U} .
$$

The single control vector case comes from $u_{2}=0$.

Since $S O(3, \mathbb{R})$ is a topological compact manifold, it turns out that $L A R C$ is equivalent to the controllability property [19]. Thus, in both cases, we have

$$
\Sigma_{G} \text { is controllable } \Leftrightarrow \operatorname{Span}_{\mathcal{L A}}\{\mathcal{X}, P, Q\}=\mathfrak{s o}(3, \mathbb{R}) .
$$




\subsubsection{The Non-Compact Semi-Simple Structure}

Here, we follow the reference [17]. The Iwasawa decomposition of

$$
\mathfrak{s l}(2 ; \mathbb{R})=\mathfrak{k} \oplus \mathfrak{a} \oplus \mathfrak{n}
$$

is given by

$\mathfrak{k}=\operatorname{Span}\left\{X=\left(\begin{array}{cc}0 & 1 \\ -1 & 0\end{array}\right)\right\}, \mathfrak{a}=\operatorname{Span}\left\{Y=\left(\begin{array}{cc}1 & 0 \\ 0 & -1\end{array}\right)\right\}, \mathfrak{n}=\operatorname{Span}\left\{Z=\left(\begin{array}{ll}0 & 1 \\ 0 & 0\end{array}\right)\right\}$,

with brackets $[X, Y]=2 Z-4 X,[X, Z]=Y,[Y, Z]=2 Z$.

According to this data, through the exponential map, the Lie group decomposes as the product $G=K A N$, where

$K=\left\{\left(\begin{array}{c}\cos \theta \\ -\sin \theta\end{array}\right.\right.$

$\left.\left.\begin{array}{l}\sin \theta \\ \cos \theta\end{array}\right): 0 \leq \theta<2 \pi\right\}, A=\left\{\left(\begin{array}{cc}x & 0 \\ 0 & \frac{1}{x}\end{array}\right): x>0\right\}, N=\left\{\left(\begin{array}{ll}1 & y \\ 0 & 1\end{array}\right): y \in \mathbb{R}\right\}$

is the compact, Abelian and nilpotent part of $G$, respectively. Furthermore, any element in $G$ has the form $g=(\theta, x, y)$.

Any $U=X, Y, Z$ give raise a linear vector field as follows,

$$
\mathcal{D}=-a d(U) \Longrightarrow \mathcal{X}_{g}=g U-U g
$$

About the controllability, we have here some results for local controllability on the identity element, some controllability relationship between the linear systems and an associated invariant system [19]. Finally, we describe a result that appear very recently involving the notion of a control set, which roughly means a region where the system is controllable in its interior. In addition, the linear system will be controllable if this set is positive invariant.

Theorem 10 ([17]). For a linear system on a semisimple Lie group G with the LARC property, we have

1. $\exists$ a control set $\mathcal{C}$ with nonempty interior around $e$ given by,

$$
\mathcal{C}=\operatorname{cl}(\mathcal{A}) \cap \mathcal{A}^{*}
$$

2. The only possible invariant control set is the whole group, i.e.,

$$
\text { if } \mathcal{C} \text { is positive invariant, then } \mathcal{C}=G \text {. }
$$

Thus, the system $\Sigma_{G}$ is controllable.

However, in this case, there is not a complete characterization for the controllability of $\Sigma_{G}$.

\section{The Pontryagin Maximum Principle}

The Pontryagin Maximum Principle is one of the main theoretical and useful optimal results available in the literature. Lev Pontryagin, a theoretical topological Mathematician, publish this result in his book Theory of Optimal Processes [40], in collaboration with several researchers of the Steklov Institute in Russia. For his work during the period from 1956 to 1961, he was awarded the Lenin Prize. The principle is fundamental to the control system theory and its applications. However, it also is instrumental for Carnot-Caratheodory geometry, sub-Riemannian, and almost-Riemannian geometries with applications in several areas. The mathematical machinery to establish and prove these results strongly depends on the associated Hamiltonian equations, obtained from a geometric point of view by lifting the initial system to the co-tangent bundle of the state manifold [26]. 
The Principle works on the co-tangent bundle $T^{*} M$ of the manifold state $M$. Our case is quite favorable since $T^{*} G$ is a trivial bundle. Precisely,

$$
T^{*} G \cong \mathfrak{g}^{*} \times G,
$$

where $\mathfrak{g}^{*}$ denotes the dual of the Lie algebra $\mathfrak{g}$ of the connected group $G$. To establish this Principle on $\Sigma_{G}$, we recall the Hamiltonian equations associated to $\Sigma_{G}$, as appear in [3,4].

For a given admissible control $u \in \mathcal{U}$, the associated $\Sigma_{G}$-Hamiltonian $H_{u}$ defined on $\mathfrak{g}^{*} \times G$ reads as

$$
H_{u}(\lambda, g)=\langle\lambda, F(g)\rangle+\sum_{j=1}^{m}\left\langle\lambda, Y^{j}\right\rangle>, g \in G,
$$

where $\lambda: \mathfrak{g} \longrightarrow \mathbb{R}, F(g)=\left(d L_{g^{-1}}\right)_{g}\left(\mathcal{X}_{g}\right)$, and $Y^{j}=\left(d L_{g}\right)_{g^{-1}} Y_{g}^{j}$.

Just observe that the system was translated from $g \in G$ to the identity.

Next, we introduce the Pontryagin Maximum Principle for the class of linear control systems on a matrix Lie group.

Theorem 11 ([4]). Let $u^{*}:[0, T] \longmapsto \Omega \subset \mathbb{R}^{m}$ be an admissible control such that the solution $\varphi\left(, u^{*}, t\right)$ of $\Sigma_{G}$ minimizes the time among all $\Sigma_{G}$-admissible curves sending $\varphi(, u, 0)$ to $\varphi(, u, T)$. Then, there exists a Lipschitzian curve $(\lambda(t), g(t)) \in T^{*} G$ such that

1. $\lambda(t) \neq 0$ for all $t \in[0, T]$. In addition, for almost all $t \in[0, T]$

2. $H_{u *}(\lambda(t), g(t))=\max _{u \in \mathcal{U}} H_{u}(\lambda(t), g(t))$

3. $H_{u *}(\lambda(t), g(t)) \geq 0$.

Furthermore, $(\lambda(t), g(t)) \in \mathfrak{g}^{*} \times G$ satisfy the Hamiltonian equations

$$
\dot{g}=\mathcal{X}_{g}+\sum_{j=1}^{m} u_{j} Y^{j}(g) \text { and } \dot{\lambda}=\lambda \circ\left(-\mathcal{D}+\sum_{j=1}^{m} u_{j} a d\left(Y^{j}\right)\right.
$$

Here, $-\mathcal{D}$ and $a d\left(Y^{j}\right)=\left[Y^{j},\right]$ are the lifting vector fields from $G$ to $T^{*} G$ of the corresponding dynamic $\mathcal{X}$ and $Y^{j}, j=1, \ldots, m$. In the general case of a system $\Sigma_{M}$ on a differentiable manifold $M$, this construction depends on a differentiable nondegenerate 2-form which always exists on $T^{*} M$. The second relationship is a differential equation on $\mathfrak{g}^{*}$ induced by $(1+m)$ derivations.

Next, we collect the main results we know about the time optimal problem for $\Sigma_{G}$, as appears in [4]. Let $\Sigma_{G}$ be a linear control system, which includes the constant control $u \in \Omega \in \mathbb{R}^{m}$.

We consider first the unbounded case. Since $H_{u}$ is maximum, then $\left\langle\lambda, Y^{j}\right\rangle=0$, for $j=1, \ldots, m$. Moreover, if $(\lambda(t), g(t))$ satisfy the Pontryagin Maximum Principle, it follows that,

$$
\left\langle\dot{\lambda}(t), Y^{j}\right\rangle=0 \text {, and }\left\langle\lambda(t),-D Y^{j}+\sum_{i=1}^{m} u_{i}\left[Y^{i}, Y^{j}\right]\right\rangle=0, \quad \text { a.e., } j=1, \ldots, m \text {. }
$$

For the bounded case, let $B>0$, and $-B \leq u_{j} \leq B$ for $j=1, \ldots, m$. Since $H_{u}$ is maximum, it turns out that

$$
u_{j}=\operatorname{sign}\left\langle\lambda, Y^{j}\right\rangle B, \text { if }\left\langle\lambda, Y^{j}\right\rangle \neq 0,
$$

Otherwise, $u_{j}$ is not determined. However, according to Filippov's Theorem in [26], minimizers exist. Moreover, if $\left[Y^{i}, Y^{j}\right]=0$ for $i, j=1, \ldots, m$, then we get an extra equation $\left\langle\lambda(t), D Y^{j}\right\rangle=0$. In the classical case $\Sigma_{\mathbb{R}^{n}}, a d\left(b_{j}\right)=0$, since the Lie algebra $\mathbb{R}^{n}$ is Abelian. However, in groups, it is not the case. In particular, the bang bang control theorem for $\Sigma_{\mathbb{R}^{n}}$, is not longer true for $\Sigma_{G}$, see [4]. 


\section{Examples}

In this section, we develop several examples on different groups of dimensions 2 and 3. We start with the more famous one: Stop a train in a station in minimum time, coming from the Pontryagin book, [40].

Example 1. Let us consider a train $\Gamma$ of mass one moving on the real line without friction. Denote by $x(t)$ the distance from $\Gamma$ to the origin (the station) at time $t$. From the Newton law, we have $\dot{x}(t)=y(t)$ and $\dot{y}(t)=u(t)$. Here, $u \in \mathcal{U}_{\Omega}$ with $\Omega=[-1.1] \subset \mathbb{R}$. Thus, we obtain a classical control system on the Abelian Lie group $\mathbb{R}^{2}$, as follows

$$
\Sigma_{G}:\left(\begin{array}{l}
x(t) \\
y(t)
\end{array}\right)=\left(\begin{array}{ll}
0 & 1 \\
0 & 0
\end{array}\right)\left(\begin{array}{l}
x(t) \\
y(t)
\end{array}\right)+u(t)\left(\begin{array}{l}
0 \\
1
\end{array}\right), u \in \mathcal{U}_{\Omega}
$$

Any control $u$ determine an ordinary differential equation. Geometrically, any initial condition $(x, y)$ should be steer to $(0,0)$ in minimum time. The system is restricted, satisfy the Kalman rank condition, $\operatorname{rank}\left(\begin{array}{cc}0 & 1 \\ 1 & 0\end{array}\right)=2$ and $\operatorname{Spec}(A)_{L y}=\{0\}$. Hence, according to Theorem 2, the system is controllable.

From the Pontryagin Maximum Principle, we should consider two class of controls: $u \equiv 1$ and $u \equiv-1$ and the minimum time curve is built with at most one change of the control.

The family of parabolas generated by the solutions of the differential equations

$$
\dot{x}(t)=y(t), y(t)=-1 \text { and } \dot{x}(t)=y(t), y(t)=1
$$

generates a curve built through two specific parabolas reaching the origin: $\phi^{-}$with control -1 and $\phi^{+}$with control 1 . Hence, starting from any arbitrary initial condition $\left(x_{0}, y_{0}\right)$ outside this curves, you choice the unique parabola which starting from $\left(x_{0}, y_{0}\right)$ and moving in positive time hit one of the curves $\phi^{-}$or $\phi^{+}$. After that, you change the control by remaining in the hitting curve up to reach the target. For instance, if you start from $\left(x_{0}, y_{0}\right)$ in the third quadrant and under $\phi^{-}$, you first take the integral curve $\gamma\left(t,\left(x_{0}, y_{0}\right), u \equiv 1\right)$, which means that you accelerate at maximum up to intersect $\phi^{-}$. After that, you follow the $\phi^{-}$trajectory by breaking at maximum and finishing in the origin. Just observe that the projection in the first variable

$$
\pi_{1}\left(\gamma\left(t,\left(x_{0}, y_{0}\right), u \equiv 1\right) \cap \phi^{-}\right) \in \mathbb{R}
$$

give you the distance to the station where you need to change the control.

Example 2. In the 2-dimensional connected affine group $A f f_{+}(2)$, we consider the linear system,

$$
\Sigma_{A f f_{+}(2)}: \quad\left\{\begin{array}{l}
\dot{x}=u x \\
\dot{y}=x-1
\end{array}, u \in \mathcal{U} .\right.
$$

According to Theorem, the systems is controllable. Here,

$$
D=\left(\begin{array}{ll}
0 & 0 \\
1 & 0
\end{array}\right), X=\left(\begin{array}{l}
1 \\
0
\end{array}\right), \text { and } \mathcal{X}_{g}=\left(\begin{array}{cc}
0 & x-1 \\
0 & 0
\end{array}\right)
$$

The Hamiltonian reads,

$$
\mathcal{H}\left(\lambda_{g}, g, u\right)=\left\langle\lambda, F_{g}\right\rangle+u\langle\lambda, X\rangle
$$


For the unbounded case, we obtain $\langle\lambda(t), X\rangle=0$. Thus,

$$
\langle\dot{\lambda}(t), X\rangle=\langle\lambda(t),-D X+u[X, X]\rangle=\langle\lambda(t),-Y\rangle=0 .
$$

Since, $\lambda$ is null on a basis of the Lie algebra $a f f_{+}(2)$ of the group, it follows that $\lambda=0$. It turns out that no extremal trajectory exists. Actually, in [4] the authors show that the minimal time to reach $(1,-1)$ from the identity is 1 but there is not a control $u \in \mathcal{U}$ which connect the states in 1 unit of time.

Next, we analyze the bounded case, $u \in[-1,1]$. In coordinates $\lambda_{g}=(p, q)$ the Hamiltonian is given by (5) as

$$
\mathcal{H}\left(\lambda_{g}, g, u\right)=\left\langle\lambda_{g}, \mathcal{X}_{g}\right\rangle+u\left\langle\lambda_{g}, X_{g}\right\rangle=p u x+q(x-1) .
$$

Therefore, the Hamiltonian equations are:

$$
\left\{\begin{array} { l } 
{ \dot { x } = \frac { \partial \mathcal { H } } { \partial p } = u x } \\
{ \dot { y } = \frac { \partial \mathcal { H } } { \partial q } = x - 1 }
\end{array} \text { and } \left\{\begin{array}{l}
\dot{p}=-\frac{\partial \mathcal{H}}{\partial x}=-u p-q \\
\dot{q}=-\frac{\partial \mathcal{H}}{\partial y}=0
\end{array}\right.\right.
$$

First of all, $q$ is constant. Now, if for some $t_{0}: p\left(t_{0}\right)=0$, then $q=q_{0} \neq 0$. In fact, by the Pontryagin Maximum Principle $\left(p(t), q_{0}\right)$ vanishes nowhere. Since $\dot{p}\left(t_{0}\right)=-q_{0} \neq 0$. It turns out that an optimal control takes the constant value $B$ or $-B$ and changes at most once. In fact, $p$ cannot vanish more than once.

In [15] the authors prove that the system is controllable by showing explicitly the trajectories connecting any two sates. Therefore, let us show how to reach the state $(e, 1)$ from the identity element by an optimal path. According to their computations, the solution starting at any initial condition $(x, y) \in G$, is given by concatenation of the following two flows

$$
\begin{aligned}
& \phi((x, y), u, t)=\left(e^{u t} x, \frac{\left(e^{u t}-1\right) x}{u}-t+y\right), t \in \mathbb{R}, u \neq 0 \\
& \phi((x, y), 0, t)=(x,(x-1) t+y), t \in \mathbb{R}, u=0 .
\end{aligned}
$$

In order to travel to the right side from $(1,0)$, we need to consider first the control $u=1$. We get,

$$
\phi((1,0), 1, t)=\left(e^{t},\left(e^{t}-1\right)-t\right), t \in \mathbb{R} .
$$

When, $t=1$, the state of the curve is $(e, e-2)$. However, the second coordinate does not coincide with our target. Therefore, the optimal control should change from $u \equiv 1$ to $u \equiv-1$, in some instant $t_{0}$ of the curve. Thus, we need to apply the control $u \equiv-1$, to the initial state $\left(e^{t_{0}},\left(e^{t_{0}}-1\right)-t_{0}\right)$. We obtain,

$$
\phi\left(\left(e^{t_{0}},\left(e^{t_{0}}-1\right)-t_{0}\right),-1, t\right)=\left(e^{-t} e^{t_{0}},\left(1-e^{-t}\right) e^{t_{0}}-t+\left(e^{t_{0}}-1\right)-t_{0}\right) .
$$

It turns out that

$$
-t+t_{0}=1 \text { and } e^{t_{0}}-e-t+e^{t_{0}}-1-t_{0}=1 .
$$

From that, we get

$$
e^{t_{0}}-t_{0}=\frac{1+e}{2} .
$$

The continuous function $f(x)=e^{x}-x$, satisfy $f\left(\frac{1}{2}\right)<\frac{1+e}{2}<f(2)$. Since, $f$ is strict increasing the only one switch time $t_{0}$ exists. The optimal curve is given by the optimal control $u^{*}(\zeta)=\left\{\begin{array}{c}1 \text { if } 0<\zeta \leq t_{0} \\ -1 \text { if } t_{0}<\zeta\end{array}\right\}$ and the optimal time is $t=t_{0}-1$ 
Example 3. For a 3-dimensional semisimple compact group $G=S O(3, \mathbb{R})$, we consider the unbounded linear system

$$
\Sigma_{S O(3, \mathbb{R})}: \quad \dot{g}=\mathcal{X}_{g}+u_{1} X_{g}+u_{2} Y_{g}, \text { where } D=-a d(X) .
$$

Since $\operatorname{Span}_{L A}\{\mathcal{X}, X, Y\}=\mathfrak{g}$ the system satisfies the LARC condition. As we know, $\Sigma$ is controllable.

The associated Hamiltonian function reads as

$$
\mathcal{H}\left(\lambda_{g}, g, u\right)=\left\langle\lambda, F_{g}\right\rangle+u_{1}\langle\lambda, X\rangle+u_{2}\langle\lambda, Y\rangle .
$$

The maximization condition implies $\langle\lambda(t), X\rangle=\langle\lambda(t), Y\rangle=0$. According to (6),

$$
0=\langle\dot{\lambda}(t), X\rangle=\left\langle\lambda(t),\left(-D X+u_{1} a d(X)+u_{2} a d(Y)\right) X\right\rangle=\left\langle\lambda(t),-u_{2} Z\right\rangle,
$$

In the same way,

$$
0=\langle\dot{\lambda}(t), Y\rangle=\left\langle\lambda(t),-D Y+u_{1} Z\right\rangle=\left\langle\lambda(t),\left(u_{1}-1\right) Z\right\rangle .
$$

According to the Pontryagin Maximum Principle, if a time optimal minimizer exists, then $\langle\lambda(t), Z\rangle \neq 0$. It turns out that $u_{1} \equiv 1$, and $u_{2} \equiv 0$. So, the only existent minimizers connect two points on the same integral curves of the vector field

$$
\dot{g}=\mathcal{X}_{g}+X_{g}
$$

In order to invite the reader to work out an optimal problem of a linear system $\Sigma_{G}$, on a 3-dimensional Lie group, we give a matrix representation of the solvable Lie algebras $\mathfrak{r}_{2}$ and $\mathfrak{e}$.

1. For $\mathfrak{r}_{2}$ consider the basis

$$
X=M(1,1), Y=M(2,3), Z=M(1,3) .
$$

With the change of variable $Y$ by $Z$, we obtain $\mathfrak{r}_{2}$ with the same bracket rules: $[X, Y]=$ $Y,[X, Z]=0$. The left invariant vector fields are given by

$$
X_{g}=-y \frac{\partial}{\partial x}, Y_{g}=-y \frac{\partial}{\partial y}, Z_{g}=\frac{\partial}{\partial z} .
$$

the associated simply connected matrix Lie group $G$ reads as

$$
G=\left\{g=\left(\begin{array}{ccc}
-y & 0 & x \\
0 & 1 & z \\
0 & 0 & 1
\end{array}\right): x, z \in \mathbb{R}, y<0 .\right\} \longleftrightarrow(x, y, z) \in \mathbb{R} \times \mathbb{R}_{-} \times \mathbb{R} .
$$

with the solvable structure

$$
\left(x_{1}, y_{1}, z_{1}\right) *\left(x_{2}, y_{2}, z_{2}\right)=\left(x_{1}-y_{1} x_{2},-y_{1} y_{2}, z_{1}+z_{2}\right) \text {. }
$$

The derivation Lie algebra has dimension 4 . Precisely, each derivation

$$
\mathcal{D}=\left(\begin{array}{lll}
a & b & 0 \\
0 & 0 & 0 \\
0 & c & d
\end{array}\right): a, b, c, d \in \mathbb{R}
$$

determines the linear vector field

$$
\mathcal{X}_{g}=(a x+b y+b) \frac{\partial}{\partial x}+(d z-c \ln (-y)) \frac{\partial}{\partial z} .
$$


2. Consider the Lie algebra with a basis

$$
X=M(1,3), Y=M(2,3), Z=M(1,2)-M(2,1),
$$

and brackets $[X, Y]=Z,[X, Z]=-Y$. With the change of variable $X$ by $Y$ and $Y$ by $Z$, we recover $\mathfrak{e}$.

The associated group is the so called Euclidean group of the affine transformations of $\mathbb{R}^{2}$, which preserves the Euclidean metric. It is isomorphic to the order three matrices,

$$
E=\left\{\left(\begin{array}{cc}
P & x \\
0 & 1
\end{array}\right): P P^{T}=I d, x \in \mathbb{R}^{2}\right\} .
$$

This realization is geometrical. The orthogonal matrix $P$ and the vector $x \in \mathbb{R}^{2}$ represents a rotation and translation in the plane, respectively. Again, any derivation is inner, and each linear vector field is associated with $\mathcal{D}=-a d(U), U \in \mathfrak{e}$.

\section{Conclusions}

In this article, we describe some existent information of the class of Linear Control Systems $\Sigma_{G}$ on a low dimensional matrix Lie group $G$. As a second purpose, we invite the Mathematical community to consider real-world applications of the Pontryagin Maximum Principle and the Equivalence Theorem for $\Sigma_{G}$. Also, we challenge some theoretical open problems.

Specifically, we inform about the controllability property; the existence, uniqueness and the shape of control sets, with and without an empty interior. Furthermore, we establish the Pontryagin Maximum Principle for $\Sigma_{G}$. We mainly focus on Lie groups of dimension two and three.

Through several examples, we explicitly show how to compute a linear system on a matrix group. We hope the Mathematical community will have the opportunity to take profit of this construction and apply this theory on real world situations.

Through the text we also mention some open problems for $\Sigma_{G}$. For instance,

1. To compute the control sets when $G$ has dimension three

2. To characterize the controllability property when $G=S L(2, \mathbb{R})$

3. To study the optimal control problem with quadratic cost.

Author Contributions: W.V., V.A. and M.T. participated in the concepcion and desisgn of the article. Drafting and revising were mainly doing by V.A. and M.T. All authors have read and agreed to the published version of the manuscript.

Funding: We thanks Universidad Nacional de San Agustín de Arequipa, UNSA, Arequipa, Perú. This article was supported by the Research Project under the Contract IBA-IB-04-2020-UNSA.

Institutional Review Board Statement: Not applicable.

Informed Consent Statement: Informed consent was obtained from all subjects involved in the study.

Data Availability Statement: The data presented in this study are available in the mentioned references.

Conflicts of Interest: The authors declare no conflict of interest.

\section{References}

1. Brockett, R.W. Finite Dimensional Linear Systems; John Wiley \& Sons: Hoboken, NJ, USA, 1970; ISBN 978-0-471-10585-5.

2. Jurdjevic, V. Geometric Control Theory; Cambridge University Press: Cambridge, UK, 1997.

3. Ayala, V.; Jouan, P. Almost Riemannian Structures and Linear Control Systems on Lie Groups. SIAM J. Control Optim. 2016, 54, 2919-2947. . [CrossRef]

4. Ayala, V.; Juan, P.; Torreblanca, M.; Zsigmond, G. Time Optimal Control for Linear Systems on Lie Groups; Submitted to Systems and Control Letters; Elsevier: Amsterdam, The Netherlands, 2020. 
5. Aseev, S.M.; Kryazhimskii, A.V. The Pontryagin maximum principle and optimal economic growth problems. Proc. Steklov Inst. Math. 2007, 257, 1-255. [CrossRef]

6. Reeds, J.A.; Sheep, L.A. Optimal paths for a car that goes both forwards and backwards. Pac. J. Math. 1990, 145, 367-393. [CrossRef]

7. Ledzewick, U.; Shattler, H. Optimal controls for a two compartment model for cancer chemoterapy. Optim. Theory Appl. JOTA 2002, 114, 241-246.

8. Zamardzija, N. Qualitative and Control Behavior of a Class of Chemical and Biological Systems. IFAC Proceeding Vol. 1989, 22, 351-355. [CrossRef]

9. Byrnes, C.; Isidori, A. On the Attitude Stabilization of Rigid Spacecraft. Automatica 1991, 27, 87-95. [CrossRef]

10. El-Osery, A. Control and Systems Engineering A Report on Four Decades of Contributions. In Studies in Systems, Decision and Control; Springer International Publishing: Cham, Switzerland, 2015.

11. Mittenhuber, D. Dubin's Problem in the Hyperbolic Space. Geometric Control and Non-Holonomic Mechanics. In CMS Conference Proceedings; Jurdjevic, V., Sharpe, R.W., Eds. American Mathematical Society: Providence, RI, USA, 1998.

12. Sussmann, H.J.; Willems, J. Three Centuries of Curves Minimization: From the Brachistochrone to the Maximum Principle. IEEE Control Syst. Mag. 1997, 17, 32-44. [CrossRef]

13. Noether, E. Invariant Variation Problems. Transp. Theory Stat. Phys. 1971, 1, 186-207. [CrossRef]

14. Jouan, P. Equivalence of Control Systems with Linear Systems on Lie Groups and Homogeneous Spaces. ESAIM Control Optim. Calc. Var. 2010, 16, 956-973. [CrossRef]

15. Ayala, V.; Silva, A.D. The control set of a linear control system on the two dimensional Lie group. J. Differ. 2020, $268,6683-6701$. [CrossRef]

16. Ayala, V.; Silva, A.D. On the characterization of the controllability property for linear control systems on nonnilpotent, solvable three-dimensional Lie groups. J. Differ. Equ. 2019, 266, 8233-8257. [CrossRef]

17. Ayala, V.; Silva, A.D.; Jouan, P.; Zsigmond, G. Control sets of linear systems on semi-simple Lie groups. J. Differ. Equ. 2020, 269, 449-466. [CrossRef]

18. Ayala, V.; Silva, A.D. Controllability of Linear Control Systems on Lie Groups with Semisimple Finite Center. SIAM J. Control Optim. 2017, 55, 1332-1343. . [CrossRef]

19. Ayala, V.; Martín, L.S. Controllability Properties of a Class of Control Systems on Lie Groups. Lect. Notes Control Inf. Sci. 2001, 1, 83-92.

20. Ayala, V.; Tirao, J. Linear control systems on Lie groups and Controllability. Am. Math. Soc. Ser. Pure Math. 1999, $64,47-64$.

21. Ayala, V.; Todco, M.T. Boundedness of control sets of Linear control systems. Open Math. 2018, 2018, 1-10. .

22. Silva, A.D. Controllability of linear systems on solvable Lie groups. SIAM J. Control Optim. 2016, 54, 372-390. [CrossRef]

23. Jouan, P. Controllability of linear systems on Lie groups. J. Dyn. Control Syst. 2011, 17, 591-616. [CrossRef]

24. Jouan, P.; Dath, M. Controllability of Linear Systems on low dimensional Nilpotent and Solvable Lie groups. J. Dyn. Control Syst. 2016, 22, 207-225.

25. Markus, L. Controllability of multi-trajectories on Lie groups. In Dynamical Systems and Turbulence, Warwick; Lecture Notes in Mathematics; Springer: Berlin/Heidelberg, Germany, 1980; Volume 898, pp. 250-265.

26. Agrachev, A.A.; Sachkov, Y.L. Control Theory from the Geometric Viewpoint; Encyclopaedia of Mathematical Sciences; Control Theory and Optimization II; Springer: Berlin, Germany, 2004; Volume 87.

27. Colonius, F.; Kliemann, W. The Dynamics of Control; Birkhauser: Basel, Switzerland, 2000.

28. Ioffe, A.; Malanowsky, K.; Trolstzsch, F. "50 Years of Optimal Control”, Special Issue, of Control and Cybernetics, 2009/4. Available online: http:/ / control.ibspan.waw.pl:3000/contents/list?year=2009 (accessed on 20 October 2020).

29. Whonam, M. Linear Multivariable Control: A Geometric Approach; Springer: New York, NY, USA, 1979; Volume 29; pp. 321-331.

30. Boothby, W. An Introduction to Differentiable Manifolds and Riemannian Geometry, 2nd ed.; ser. Pure and Applied Mathematics; Academic Press: Boston, FL, USA, 1986.

31. Elliott, D.L. Bilinear Control Systems: Matrices in Action; Springer: Berlin/Heidelberg, Germany, 2009.

32. Isidori, A. Nonlinear Control Systems; Springer: London, UK, 1989; ISBN 3-540-19916-0.

33. Helgason, S. Differential Geometry, Lie Groups and Symmetric Spaces; Academic Press: Cambridge, MA, USA, 1978.

34. Onishchik, A.L.; Vinberg, E.B. Lie Groups and Lie Algebras III-Structure of Lie Groups and Lie Algebras; Springer: Berlin, Germany, 1994.

35. Martin, L.A.B.S. Algebras de Lie, 2nd ed.; Ed. Unicamp: Campinas, Brazil, 2010.

36. Varadarajan, V. Lie Groups, Lie Algebras, and Their Representations; Springer: New York, NY, USA, 1984.

37. Agrachev, A.A.; Boscain, U.; Charlot, G.; Ghezzi, R.; Sigalotti, M. Two-dimensional almost-Riemannian structures with tangency points. Ann. Inst. H. Poincare Anal. Non Lineaire 2010, 27, 793-807. [CrossRef]

38. Sachkov, Y. Control Theory on Lie Groups. J. Math. Sci. 2009, 156, 381. [CrossRef]

39. Sachkov, Y. Survey on Controllability of Invariant Control Systems on Solvable Lie Groups. AMS Proc. Symp. Pure Math. 1999, 64, 297-317.

40. Pontryagin, L.S.; Boltyanskii, V.G.; Gamkrelidze, R.V.; Mishchenko, E.F. The Mathematical Theory of Optimal Processes; Interscience Publishers John Wiley \& Sons, Inc.: New York, NY, USA; London, UK, 1962. 